\title{
Anti-maloperation Study of Zero-sequence Directional Protection for Three Circuits on the Same Tower
}

\author{
Yaru Dong ${ }^{1, a}$, Hui Huang ${ }^{1, b}$, Jieru Dong ${ }^{2, c}$ \\ ${ }^{1}$ School of Electrical Engineering, Beijing Jiao tong University, Beijing 100044, China; \\ 2 State Grid Xin Zhou Power Supply Company, Xin Zhou 034000, China. \\ adongyaru@bjtu.edu.cn, bhhuang@bjtu.edu.cn, cjierudong@126.com
}

Keywords: three circuits on the same tower, zero-sequence directional protection, zero-sequence voltage compensation, zero-sequence criterion, negative-sequence criterion

\begin{abstract}
Based on the limitation of zero-sequence voltage compensation of traditional zero-sequence directional protection principle, an improved anti-maloperation protection strategy is proposed. First of all, by analyzing the characteristics of zero-sequence component, zero-sequence protection principle and maloperation reason of three circuits on the same tower, it is found that zero-sequence voltage over-compensation will change the polarity of zero-sequence voltage and lead to protection maloperation. Considering that the negative-sequence components are not affected by the mutual inductance between lines, a method of introducing negative-sequence components and reducing compensation impedance is proposed, which can reduce the possibility of over-compensating greatly. Based on this, the improved zero-sequence directional protection anti-maloperation logic is constructed. When a longitudinal fault occurs on the line, this method can achieve the purpose of preventing maloperation accurately. Finally, the effectiveness and feasibility of the proposed method are verified by numerical simulation.
\end{abstract}

\section{Introduction}

At present, the zero-sequence directional protection has the advantages of high sensitivity and simple principle. And generally it is used as the main protection for high-voltage line protection[1,2]. However, there is zero-sequence mutual inductance in the three circuits on the same tower, which increases the types and complexity of faults, and may cause protection maloperation[3,4]. Finally, it directly affects the power supply reliability and stable operation of the power system.

At home and abroad, the researches on the three circuits on the same tower at present mainly focus on the calculation of line parameters and electric field. The researches on zero-sequence directional protection still stay on the same tower with double-circuit lines[5,6,7]. In contrast, there are few researches on the zero-sequence directional protection. Therefore, it is of great significance to study the anti-maloperation of zero-sequence directional protection of three circuits on same tower in the case of longitudinal fault.

Based on the analysis of the zero-sequence component characteristics in the longitudinal fault of the three circuits on the same tower, the zero-sequence voltage over compensation may be the cause of maloperation of the zero-sequence directional protection. In order to prevent the maloperation, a method of reducing the compensation impedance and introducing the criterion of negative-sequence component is present. A large number of simulation experiments are carried out to verify the feasibility of the proposed method.

\section{Zero-Sequence Component Characteristics of Longitudinal Faults}

The distributions of zero-sequence components in the case of longitudinal faults on the same tower with three circuits are shown in Fig.1. When a broken-line fault occurs in the I circuit, it is equivalent to connect a voltage source at the fault point. Under the effect of this voltage source, the zero-sequence voltage polarities of the fault line are opposite, and the zero-sequence currents passing 
through the fault line flow from the negative polarity to the positive polarity. At this moment, II circuit will be electromagnetically coupled, so that the polarities of the zero-sequence voltage at the two ends of the healthy line are opposite and the zero-sequence voltage difference becomes smaller. A zero-sequence voltage zero-crossing point will appear on the healthy line. The impact of the II circuit on the III circuit is the opposite of the effect of the I circuit.
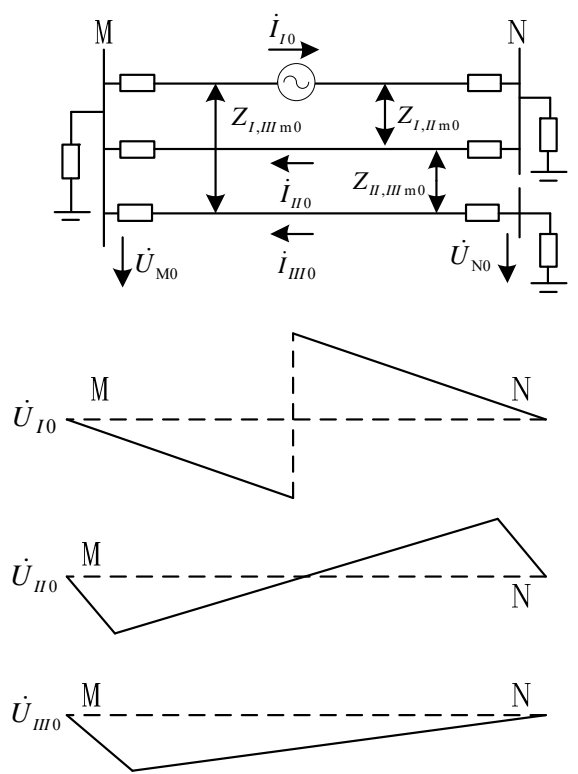

Fig.1 Distributions of zero-sequence components during I broken-line faults

\section{Zero-Sequence Directional Protection Principle and Analysis of Misoperation}

\subsection{Zero-Sequence Directional Protection Principle.}

The zero-sequence directional element judges the fault direction through the phase difference between the zero-sequence voltage phasors and the zero-sequence current phasors. The normal zero-sequence directional protection criterion can be set as:

$$
\left\{\begin{array}{l}
170^{\circ}<\arg \left(\frac{3 \dot{U}_{(0)}}{3 \dot{I}_{(0)}}\right)<350^{\circ}, \text { forward fault } \\
-10^{\circ}<\arg \left(\frac{3 \dot{U}_{(0)}}{3 \dot{I}_{(0)}}\right)<170^{\circ}, \text { reverse fault }
\end{array}\right.
$$

At the same time, the zero-sequence current start criterion also needs to be satisfied:

$$
\left|3 \dot{I}_{(0)}\right|>300 \mathrm{~A}
$$

In above formulas, $\dot{U}_{(0)}$ is the zero-sequence voltage phasor, $\dot{I}_{(0)}$ is the zero-sequence current phasor. When the fault occurs, if the zero-sequence directional elements at both ends of the line are all judged as forward faults, it can be concluded that there is a fault within the line and the breaker instantly works. But in practice, if zero-sequence voltage is too low, the accuracy of the zero-sequence directional calculation will be affected. Normally, zero-sequence voltage compensation is added to solve the problem. If the $\left|3 \dot{U}_{(0)}\right|$ is lower than $0.02 U_{N}\left(U_{N}\right.$ is the system nominal voltage), the voltage compensation should be carried out:

$$
3 \dot{U}_{(0)}^{\prime}=3 \dot{U}_{(0)}-3 \dot{I}_{(0)} Z_{\mathrm{b}}
$$

Where, the $Z_{\mathrm{b}}$ usually equals $0.7 Z_{L 0}$, which is the zero-sequence impedance of the whole line. By this compensation, we can judge the fault direction correctly. 


\subsection{Analysis of Zero-Sequence Directional Protection Operating Characteristics and Misoperation.}

When a broken-line fault occurs on I circuit, if the zero-sequence voltages at both ends of the healthy line are higher than $0.02 U_{N}$, zero-sequence directional protection does not need voltage compensation. In this case, the fault is internal disturbance, and the zero-sequence directional elements at both ends will be judged as forward faults at the same time. While the zero-sequence voltage of both ends of the II circuit are the same as that of the I circuit, and the direction of the zero-sequence current is opposite to that of the I circuit, so both ends of the line are judged as reverse faults. The polarity of the zero-sequence voltage at both ends of the III circuit are approximately the same, and the disturb point is located on the back side of the $M$ terminal. At this time, the $M$ terminal is judged as the reverse fault and the $\mathrm{N}$ terminal is judged as the forward fault, so that the protection will not malfunction.

However, if the zero-sequence voltages are below $0.02 U_{N}$, the protection will implement voltage compensation according to equation (3) and judge the fault direction with the compensated zero-sequence voltage.

When a broken-line fault occurs on I circuit, as shown in Fig.2, there is a zero-sequence voltage zero-crossing point in II circuit. Without considering the influence of mutual inductance, it is assumed that the zero-crossing point of the healthy line is located in the middle of the line. In this case, the voltage at both ends will be overcompensated, so that the zero-sequence voltage polarity are reversed. Since there is no change in the direction of the zero-sequence current, the zero sequence direction elements at both ends will be misjudged as forward fault at the same time, and the protection will be malfunctioned. Considering the effect of zero-sequence mutual inductance, the possibility of over-compensation will increase significantly at this time.

For the III circuit, the zero-sequence voltage polarity at both ends of the line are the same. As shown in Fig.2, without considering the influence of mutual inductance, after compensating, the zero-sequence voltage of $\mathrm{N}$-terminal has the same polarity and the amplitude increases, while the amplitude of M-terminal decreases. However, considering the zero-sequence mutual inductance between lines, the magnetic coupling between lines will reduce the zero-sequence voltage and increase the zero-sequence current flowing through the healthy line. In this case, the probability of over-compensation at the $\mathrm{M}$ terminal will increase significantly, then $\mathrm{M}$ terminal will be misjudged as forward fault. Since the $\mathrm{N}$ terminal is also judged as forward, the zero-sequence directional protection will malfunction.

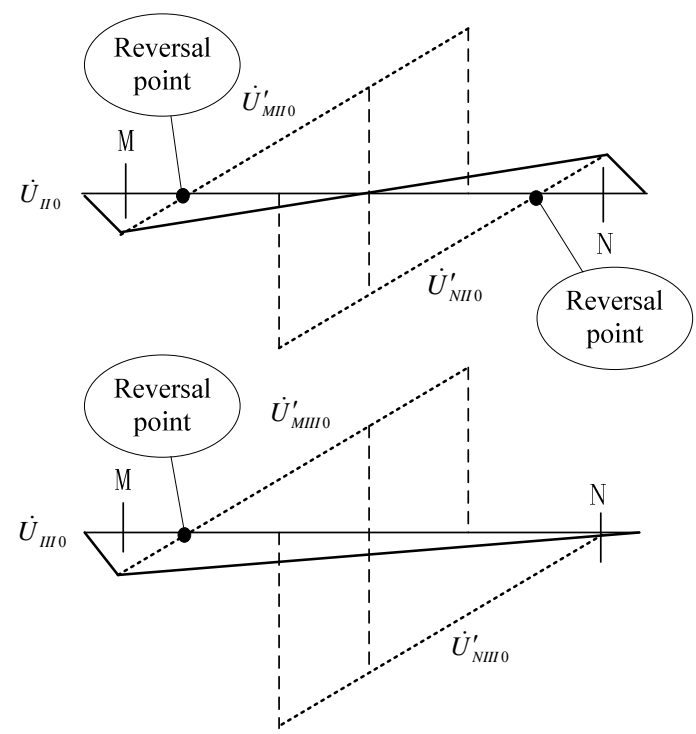

Fig.2 Voltage over compensation of zero-sequence directional element during I broken-line faults (considering mutual inductance) 


\section{Improvement of Anti-Maloperation Strategy for Zero-Sequence Directional Protection of Three Circuits on The Same Tower}

The reasons for the misoperation of zero-sequence protection of three circuits on the same tower are analyzed. This section focuses on the anti-maloperation measures. First of all, on the basis of ensuring the sensitive action of zero-sequence directional protection, we can significantly reduce the possibility of over-compensation of zero-sequence voltage by appropriately reducing the compensation impedance. Secondly, since the negative-sequence component is not affected by the mutual inductance between lines, we can introduce negative-sequence components to construct the criterion to prevent the protection from misoperation. At the same time, the negative-sequence part needs high sensitivity to avoid affecting the overall sensitivity of the improved zero-sequence directional protection in the case of internal fault.

\subsection{Improved Zero-Sequence Criterion.}

The criterion of zero-sequence directional protection is constructed in the form of phase-comparison. In the improved protection criterion, the main criterion of zero-sequence directional protection with compensation is constructed in the form of amplitude-comparison:

$$
\left|3 \dot{U}_{(0)}-3 \dot{I}_{(0)} Z_{0}\right|>\left|3 \dot{U}_{(0)}+\dot{I}_{(0)} Z_{0}\right|
$$

In above formula, $Z_{0}$ is the line zero-sequence impedance. Equation (4) is equivalent to the phase-comparison criterion which is constructed by the zero-sequence voltage compensated by $1 / 3 Z_{0}$ and the line zero-sequence current:

$$
90^{\circ}+\arg \left(\mathrm{Z}_{0}\right)<\arg \left(\frac{3 \dot{U}_{(0)}-\dot{I}_{(0)} Z_{0}}{\dot{I}_{(0)}}\right)<270^{\circ}+\arg \left(Z_{0}\right)
$$

In the meantime, in order to ensure the validity of the zero-sequence measured values involved in the calculation, the zero-sequence current start criterion also need to be satisfied:

$$
\left|3 \dot{I}_{(0)}\right|>300 \mathrm{~A}
$$

\subsection{Improved Negative-Sequence Criterion.}

First, the auxiliary criterion of negative-sequence direction with compensation is constructed.

$$
\left|3 \dot{U}_{(2)}-3 \dot{I}_{(2)} Z_{2}\right|>\left|3 \dot{U}_{(2)}+\dot{I}_{(2)} Z_{2}\right|
$$

In above formula, $\dot{U}_{(2)}$ is the negative-sequence voltage phasor, $\dot{I}_{(2)}$ is the negative-sequence current phasor. $Z_{2}$ is the line negative-sequence impedance Similarly, equation (7) is equivalent to the phase-comparison criterion which is constructed by the negative-sequence voltage compensated by $1 / 3 Z_{2}$ and the line negative-sequence current:

$$
90^{\circ}+\arg \left(Z_{2}\right)<\arg \left(\frac{3 \dot{U}_{(2)}-\dot{I}_{(2)} Z_{2}}{\dot{I}_{(2)}}\right)<270^{\circ}+\arg \left(Z_{2}\right)
$$

The introduction of negative-sequence component is to prevent misjudgment due to mutual inductance. In the broken-line fault, at least one zero-sequence directional element will not be over-compensated by $1 / 3 Z_{2}$ and can be judged as reverse to avoid the misoperation. The negative-sequence compensation voltage start criterion, negative-sequence current start criterion and negative-sequence voltage start criterion also need to be satisfied:

$$
\begin{gathered}
\left|3 \dot{U}_{(2)}-\dot{I}_{(2)} Z_{2}\right|>0.02 U_{N} \\
\left|3 \dot{I}_{(2)}\right|>150 \mathrm{~A} \\
\left|3 \dot{U}_{(2)}\right|>0.02 U_{N}
\end{gathered}
$$

Equation (11) can identify the negative-sequence voltage threshold of the weak power source. If it fails to meet the current criterion (10) when a fault occurs inside the line, the voltage criterion (11) must be satisfied. Based on this principle, we can overcome the impact of weak power sources. 


\subsection{Action Logic of the Improved Strategy}

The improved zero-sequence directional protection needs the combination of the above zero-sequence criterion and negative-sequence criterion. When a fault occurs on three circuits on the same tower, the logic of improved protection action can be shown in Fig.3.

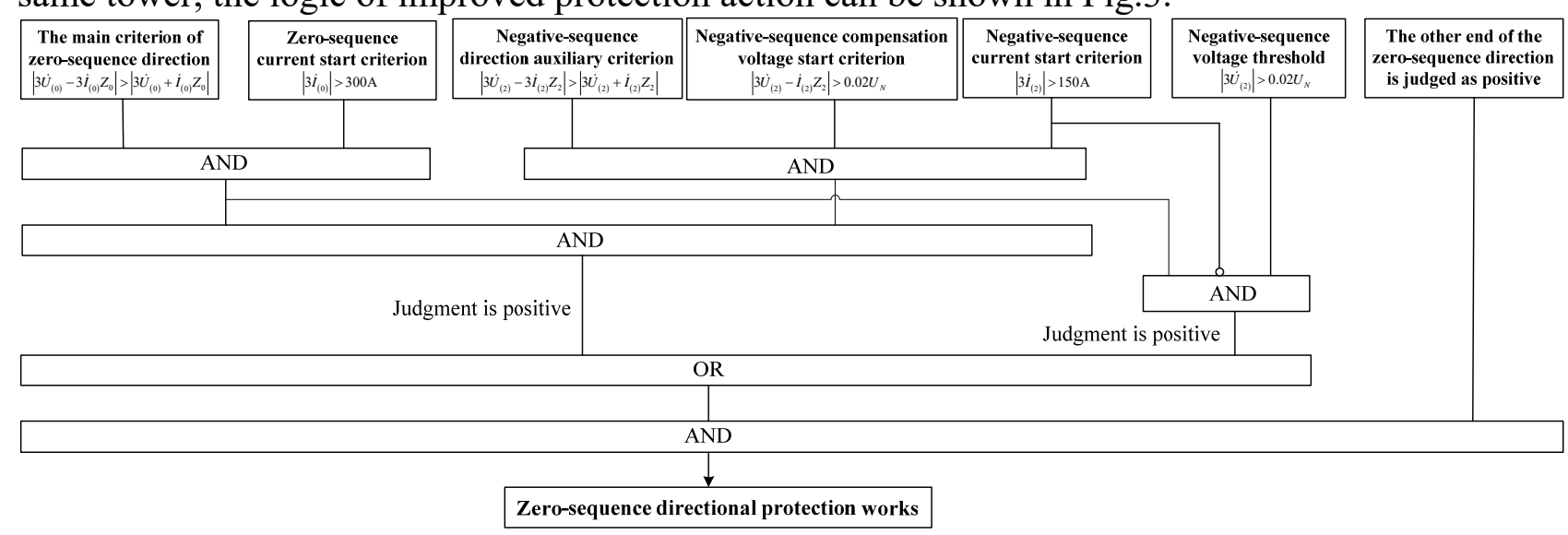

Fig.3 the logic of improved zero-sequence directional protection

\section{Simulation Analysis}

\subsection{Simulation Content.}

The structure of the simulation system is shown in Fig.4. In this system, $\dot{E}_{\mu}=500 \angle 18^{\circ} \mathrm{kV}, \dot{E}_{N}=500 \angle 0^{\circ} \mathrm{kV}, \dot{Z}_{\mu 1}=\dot{Z}_{N 1}=25 \angle 70^{\circ} \Omega, \dot{Z}_{\mu 0}=\dot{Z}_{N 0}=5 \angle 70^{\circ} \Omega, L=65 \mathrm{~km}, Z_{1}=Z_{2}=0.01$ $+j 0.359 \Omega / \mathrm{km}, Z_{0}=0.148+j 0.774 \Omega / \mathrm{km}$.

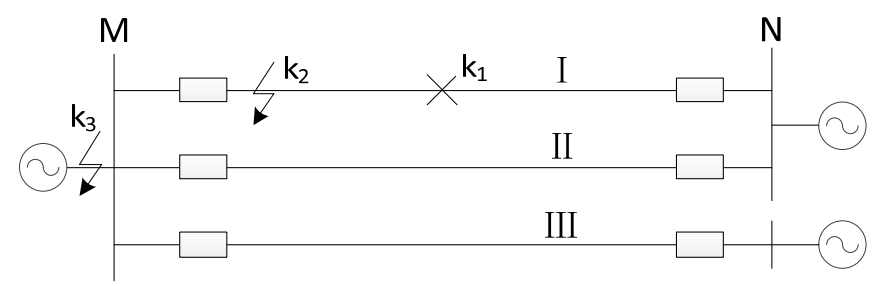

Fig.4 Simulation system

The simulation types include the broken-line faults and short-circuit faults: (1) Phase A broken-line fault occurs on k1. (2) Phase A short circuit fault occurs on k2. (3) External short circuit fault occurs on $\mathrm{k} 3$. The fault occurs at $0.2 \mathrm{~s}$.

\subsection{Simulation Results and Analysis.}

The simulation results are as follows. MI, NI represent protection elements of the I circuit. MII, NII represent protection elements of the II circuit. MIII, NIII represent protection elements of the III circuit. The judgment result " 1 " indicates forward fault, " 0 " indicates no action or reverse fault.

(1) The protection judgment results when phase A broken-line fault occurs on k1 are shown in Fig. 5. The six pictures respectively represent the action results of the directional elements at two ends of the line I, II, and III in sequence, which is the same below. It can be seen from the figure that both ends of the fault line are shown as forward fault. And both relays of the M terminals of II and III circuits are shown as reverse fault, and both relays of the $\mathrm{N}$ terminal are shown as positive fault. In this case, the improved zero-sequence directional protection does not operate on the II and III circuits, and the simulation shows that if the healthy line adopts the traditional zero-sequence directional protection, a maloperation will occur in this case. So, it can be seen that the principle of improved zero-sequence directional protection significantly improves the protection reliability. 

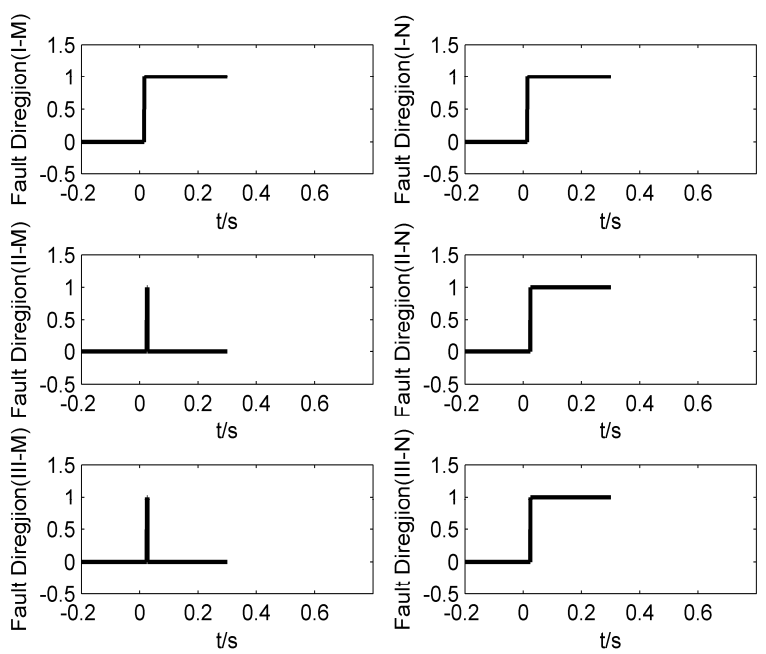

Fig. 5 Phase A broken-line fault occurs on k1.

(2) The protection judgment results when phase A short circuit fault occurs on $\mathrm{k} 2$ are shown in Fig.6. For I circuit, the fault point is located inside the line, so the zero-sequence directional elements at both ends of the line react to forward fault. It indicates that the improved zero-sequence directional protection does not affect the protection sensitivity in the internal fault. The zero-sequence directional element near the fault point of II, III circuits are shown as reverse fault, and the far are shown as positive fault. So, the protection will not malfunction. It meets the reliability requirements.
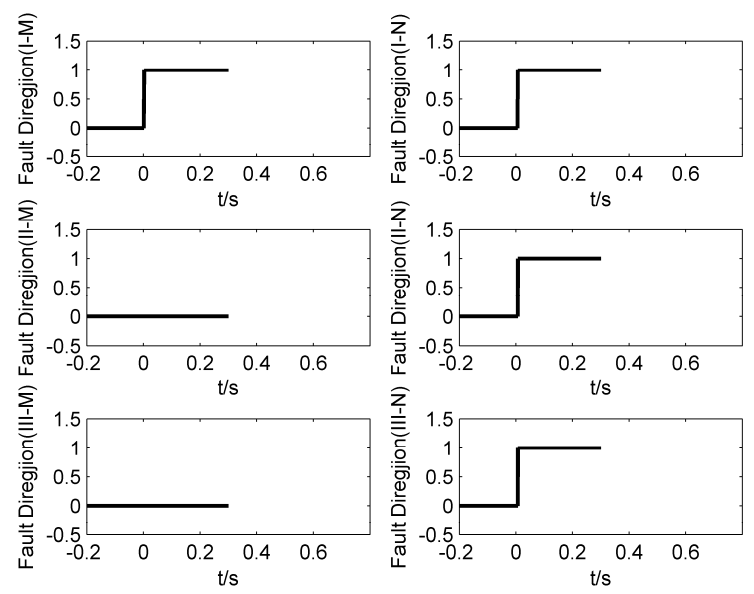

Fig. 6 Phase A short circuit fault occurs on k2

(3) The protection judgment results when external short circuit fault occurs on k3 are shown in Fig.7. There is no maloperation on I, II, III circuits, which indicates that the improved zero-sequence directional protection still has good reliability in external fault.
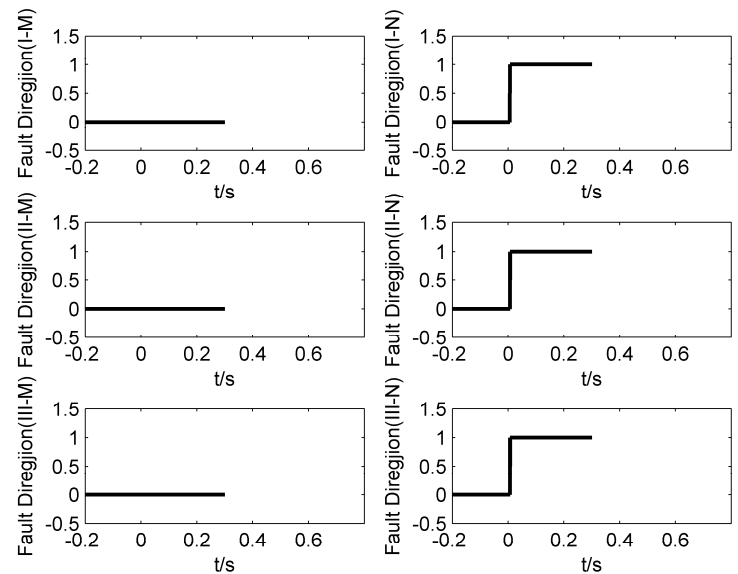

Fig.7 External short circuit fault occurs on $\mathrm{k} 3$ 
In summary, we can confirm that the improved zero-sequence directional protection has good sensitivity and reliability under the condition of different fault types and fault points and can effectively prevent the protection from malfunctioning.

\section{Conclusion}

In the three circuits on the same tower, when a longitudinal fault occurs on I circuit, the healthy line may be affected by the electromagnetic coupling of the fault line, which may cause protection maloperation by over-compensation of the zero-sequence directional elements at both ends of the line. However, the improved anti-maloperation protection strategy of zero-sequence protection overcomes the influence of mutual inductance by introducing negative-sequence components. In the case of single-phase short-circuit or broken-line faults, the adjacent healthy line will not malfunction. When the internal fault occurs, the improved zero-sequence directional protection can operate correctly without affecting the sensitivity of protection. Therefore, this strategy significantly improves the reliability of zero-sequence directional protection.

\section{References}

[1]. CHEN Wenzhe, HAO Zhiguo, ZHANG Baohui, et al. Characteristic analysis on longitudinal fault of dual-circuit transmission line and improvement of zero-sequence directional protection[J]. Electric Power Automation Equipment. Vol. 36 (2016) No. 11, p.133-139.

[2]. WANG Ping, CHEN Jiulin. Analysis of longitudinal zero sequence direction protection misoperation and its countermeasures[J]. Electric Power Automation Equipment. Vol. 27 (2007) No. 7, p. 122-125.

[3]. JIANG Xianguo, DU Dingxiang, WANG Xingguo, et al. Maloperation prevention study of zero sequence direction protection to broken-line faults of parallel circuits on same tower[C]. DRPT 2015.

[4]. ZHU Shengshi. Principle and technique of high voltage network protective relaying[M]. Beijing: China Electric Power Press, 2005, p. 270-277.

[5]. CHEN Wenzhe, HAO Zhiguo, ZHANG Baohui, et al. Characteristic analysis on longitudinal fault of dual-circuit transmission line and improvement of zero-sequence directional protection[J]. Electric Power Automation Equipment. Vol.36 (2016) No. 11, p.133-139.

[6]. CHEN Qing, XU Wenyuan. Research on fault analysis of double circuit lines on the same tower[J]. Proceedings of the CSEE. Vol.25 (2005) No. 20, p.68-71.

[7]. WU Lin-lin, HUANG Shao-feng. Effects of zero-sequence mutual inductance on zero-sequence pilot protection of parallel lines[J]. Power System Protection and Control, Vol.39(2011) No. 3, p.24-28. 